\title{
Mathematical Finance: Applications of Stochastic Process
}

\author{
${ }^{1}$ S. K. Sahoo, ${ }^{2}$ M. N. Mishra \\ ${ }^{1}$ Asst. Professor, Department of Mathematics, VITS Engineering College, Damanabhuin, NH-5, Khurda-20, \\ Odisha, India \\ 2 \\ Professor, Mathematical Finance, Institute of Mathematics \& Applications, Bhubaneswar, Odisha, India
}

Abstract: One of the momentous equations in financial mathematics is the Black-Scholes equation, a partial differential equation that governs the value of financial derivatives, such as options. In this paper, we attempt to show the application of Stochastic Process. We have shown how geometric Brownian motion \& Ito's Lemma overlaps on Option Pricing.

Key Words: - Geometric Brownian motion, Ito's Lemma, Black-Scholes Equation

\section{Introduction}

Investors pay for stocks and bonds in the monetary market, putting their funds at risk for the chance to receive a return. As the time of Phoenicians, they have sought to reduce this risk value for each level of expected return. In order to do so, a whole range financial tool have been developed, known as derivatives, assets who derive assets as of another financial asset.

The scenery of derivative assets provides an interesting means of expression for the analysis and application of Brownian motion and solving partial derivative equations, while maintaining its real world applications. Several articles have been written on modeling movements in financial markets with stochastic calculus. Possibly the most eminent of these described the Nobel Prize winning Black-Scholes option pricing model [4]. In several articles, mathematicians, specifically Robert Almgren's[5] and Anastasios Malliaris[1], have attempted to more rigorously bridge the gap between random motion and option pricing.

\subsection{Financial}

\section{Terminology}

- Asset: An object that provides a claim to future cash flows.

- Efficient Market Hypothesis: There is no opportunity for arbitrage in the market.

- Derivative: A financial asset that derives its value from another asset.

- Option: A derivative that provides the opportunity, but not obligation to buy or sell an asset at a predetermined price in the future.

- Strike Price: The predetermined price for executing an option. For a call option, if the market price rises above the strike price, the investor will be willing to buy. For a put option, if the market price falls below the strike price, the investor will want to sell the underlying asset.

\subsection{Stochastics}

- Probability Space: A construct of three components, $(\Omega, F, P)$, where

1. $\Omega$ is the set of all possible outcomes.

2. $F$ is the set of all events, where each event has zero or more outcomes.

3. $P$ is the assignment of probabilities to each event.

- With Probability 1: Also known as almost surely. The probability of an event occurring tends to 1 given some limit. Note that this differs from surely in that surely indicates that no other event is possible, while almost surely indicates that other events become less and less likely.

- A collection of sets $\mathbf{F}$ is called $\sigma$-algebra if for a sequence of sets $A_{k} \in \mathbf{F}, \cup_{1}^{\infty} A_{k} \in \mathbf{F}$ and is closed under complementation. The sets $A \in \mathbf{F}$ are $\mathbf{F}$-measurable.

- $\mathbf{M}[0, \mathrm{~T}]$ denotes the set of functions $f(t)$ such that $f(t)$ is defined on $[0, \mathrm{~T}]$, measurable with respect to the $\sigma$-algebra $\mathbf{F}_{t}$ for all $t$, and $\int_{0}^{T}|f(t)|^{2} d t$ is finite with probability 1 . 


\section{Brownian Motion}

\section{Mathematical Stochastics}

The dominion of financial asset pricing borrows a great deal from the field of stochastic calculus. The price of a stock tends to follow a Brownian motion.

Definition A stochastic process $w(t)$ is said to track a Brownian motion on $[0, \mathrm{~T}]$ if it satisfies the following:

1. $w(0)=0$.

2. $w(t)$ is almost surely continuous.

3. For arbitrary $t_{1}, t_{2}, t_{3}, \ldots, t_{n}$ where $0<t_{1}<t_{2}<\cdots<t_{n}<T$, the variables $w(0), w\left(t_{1}\right)-$ $w(0), w\left(t_{2}\right)-w\left(t_{1}\right), \ldots, w\left(t_{n}\right)-w\left(t_{n-1}\right)$ are mutually independent. In other words, it is a process with independent increments.

4. The mean (or expected) value, $\mathbf{E} w(t)$ is 0 .

5. The process $w(t)$ takes on a normal distribution density around its mean. More specifically, $\mathrm{E}\left[\mathrm{w}\left(\mathrm{t}_{\mathrm{k}+1}\right)-\right.$ $\left.\mathrm{w}\left(\mathrm{t}_{\mathrm{k}}\right)\right]^{2}=\mathrm{t}_{\mathrm{k}+1}-\mathrm{t}_{\mathrm{k}}$.

\section{The Ito Integral and the Ito Differential}

A natural response to a Brownian motion $w(t)$ is the desire to integrate with respect to it. Thus, for a function/process $f$ over a probability space $\omega$, we seek to make sense of a stochastic integral

$$
\int_{0}^{T} f(t, x) d w(t)
$$

Note that with a differential function $g(t)$, we can evaluate the Riemann Stieljes integral of

$$
\int_{0}^{T} f(t) d g(t)
$$

by using $\lim _{n \rightarrow \infty} \sum_{j=0}^{n-1} f\left(t^{*}\right)\left[g\left(t_{j+1}\right)-g\left(t_{j}\right)\right]$. Where $t^{*}$ is a point on the interval $\left[t_{j}, t_{j+1}\right]$, and the series converges to the same limit regardless of our selection of $t^{*}$. With Brownian motion, because of the independent increment quality, $w(t)$ is nowhere differentiable, and as such, we cannot evaluate the Riemann Stieljes integral (the limit of the sum is dependent on our selection of $t^{*}$ ). As a result, there are as many stochastic integrals as there are selections of $t^{*}$.

Definition The Ito integral, takes our selection of $t^{*}$ as the left endpoint, $t_{j}$. We thus have

$$
\int_{0}^{T} f(t, x) d w(t)=\lim _{n \rightarrow \infty} \sum_{j=0}^{n-1} f\left(t_{j}\right)\left[w_{t_{j+1}}-w_{t_{j}}\right]
$$

With a stochastic integral, it seems only suitable to have a stochastic differential. It is defined as follows.

Definition Suppose there exist two functions $u(t)$ and $v(t)$ in $\mathbf{M}[0, T]$ such that for all $0 \leq t_{i} \leq t_{f} \leq T$,

$$
X\left(t_{f}\right)-X\left(t_{i}\right)=\int_{t_{i}}^{t_{f}} u(t) d t+\int_{t_{i}}^{t_{f}} v(t) d w(t)
$$

Then the Ito differential of a process $X(t)$ is defined to be

$$
d X(t)=u(t) d t+v(t) d w(t)
$$

\section{Ito's Lemma}

Stochastic calculus contains an analogue to the chain rule in ordinary calculus. If a method follows geometric Brownian motion, we can relate Ito's Lemma, which states [4]:

Theorem 3.1 Suppose that the process $X(t)$ has a stochastic differential $d X(t)=u(t) d t+v(t) d w(t)$ and that the function $f(t, x)$ is nonrandom and defined for all $t$ and $x$. in addition, suppose $f$ is continuous and has continuous derivatives $f_{t}(t, x), f_{x}(t, x), f_{x x}(t, x)$. Then the stochastic process $Y(t)=f(t, X(t))$ also has a stochastic differential, and

Or in integral form

$$
d Y(t)=\left[f_{t}(t, X(t))+f_{x}(t, X(t)) u(t)+\frac{1}{2} f_{x x}(t, X(t)) v^{2}(t)\right] d t+f_{x}(t, X(t)) v(t) d w(t)
$$

$$
Y\left(t_{f}\right)-Y\left(t_{i}\right)=\int_{t_{i}}^{t_{f}}\left[f_{t}(t, X(t))+f_{x}(t, X(t)) u(t)+\frac{1}{2} f_{x x}(t, X(t)) v^{2}(t)\right] d t+\int_{t_{i}}^{t_{f}} f_{x}(t, X(t)) v(t) d w(t)
$$


This proof is borrowed largely from Gikhman and Skorokhod [3].

Proof First, let us assume that $u(t)$ and $v(t)$ are independent of $t$. Let $t_{i}=t_{0}<t_{1}<\cdots<t_{n}=t_{f}$.

Then

$Y\left(t_{f}\right)-Y\left(t_{i}\right)=f\left(t_{f}, X\left(t_{f}\right)\right)-f\left(t_{i}, X\left(t_{i}\right)\right)=\sum_{k=0}^{n-1}\left[f\left(t_{k+1}, X\left(t_{k+1}\right)\right)-f\left(t_{k}, X\left(t_{k}\right)\right)\right]$

However, note that the Taylor expansion of the summand is

$$
\begin{aligned}
f\left(t_{k+1}, X\left(t_{k+1}\right)\right) & -f\left(t_{k}, X\left(t_{k}\right)\right) \\
& =f_{t}\left(t_{k}, X\left(t_{k}\right)\right)\left(t_{k+1}-t_{k}\right)+f_{x}\left(t_{k}, X\left(t_{k}\right)\right)\left[X\left(t_{k+1}\right)-X\left(t_{k}\right)\right] \\
& +\frac{1}{2} f_{x x}\left(t_{k}, X\left(t_{k}\right)\right)\left[X\left(t_{k+1}\right)-X\left(t_{k}\right)\right]^{2}+O\left(t X, t^{2}, X^{3}\right)
\end{aligned}
$$

The $O\left(t X, t^{2}, X^{3}\right)$ will become irrelevant with probability 1 as $\max \left(t_{k+1}-t_{k}\right) \rightarrow 0$, by way of thinking similar to that in Lemma A. 1. As it turns out, we will show that the $\left[X\left(t_{k+1}\right)-X\left(t_{k}\right)\right]^{2}$ term retains an $O(t)$ term, so we keep it for now.

Because

We can substitute to get

$$
X\left(t_{k+1}\right)-X\left(t_{k}\right)=u(t)\left(t_{k+1}-t_{k}\right)+v(t)\left(w\left(t_{k+1}\right)-w\left(t_{k}\right)\right),
$$

$$
\begin{aligned}
f\left(t_{k+1}, X\left(t_{k+1}\right)\right)- & f\left(t_{k}, X\left(t_{k}\right)\right) \\
& =f_{t}\left(t_{k}, X\left(t_{k}\right)\right)\left(t_{k+1}-t_{k}\right)+f_{x}\left(t_{k}, X\left(t_{k}\right)\right) u\left(t_{k}\right)\left(t_{k+1}-t_{k}\right) \\
& +f_{x}\left(t_{k}, X\left(t_{k}\right)\right) v\left(t_{k}\right)\left[w\left(t_{k+1}\right)-w\left(t_{k}\right)\right]+\frac{u\left(t_{k}\right)^{2}}{2} f_{x x}\left(t_{k}, X\left(t_{k}\right)\right)\left(t_{k+1}-t_{k}\right)^{2} \\
& +u(t) v(t) f_{x x}\left(t_{k}, X\left(t_{k}\right)\right)\left(t_{k+1}-t_{k}\right)\left[w\left(t_{k+1}\right)-w\left(t_{k}\right)\right] \\
& +\frac{v\left(t_{k}\right)^{2}}{2} f_{x x}\left(t_{k}, X\left(t_{k}\right)\right)\left[w\left(t_{k+1}\right)-w\left(t_{k}\right)\right]^{2}
\end{aligned}
$$

As shown in Lemma A.1 (in the appendix), the terms that sum over the $\left(t_{k+1}-t_{k}\right)^{2}$ term and the $\left(t_{\mathrm{k}+1}-\right.$ $\left.\mathrm{t}_{\mathrm{k}}\right)\left[\mathrm{w}\left(\mathrm{t}_{\mathrm{k}+1}\right)-\mathrm{w}\left(\mathrm{t}_{\mathrm{k}}\right)\right]$ term will tend to 0 with probability 1 as $\max \left(t_{k+1}-t_{k}\right) \rightarrow 0$. If we now sum over the remaining terms, we have

$$
\begin{aligned}
Y\left(t_{f}\right)-Y\left(t_{i}\right)= & \max _{\max }\left[\sum_{\left.t_{k+1}-t_{k}\right) \rightarrow 0}^{n-1} f_{k=0}\left(t_{k}, X\left(t_{k}\right)\right)\left(t_{k+1}-t_{k}\right)\right. \\
& +\sum_{k=0}^{n-1} f_{x}\left(t_{k}, X\left(t_{k}\right)\right) u\left(t_{k}\right)\left(t_{k+1}-t_{k}\right) \\
& +\sum_{k=0}^{n-1} f_{x}\left(t_{k}, X\left(t_{k}\right)\right) v\left(t_{k}\right)\left[w\left(t_{k+1}\right)-w\left(t_{k}\right)\right] \\
& \left.+\frac{v\left(t_{k}\right)^{2}}{2} \sum_{k=0}^{n-1} f_{x x}\left(t_{k}, X\left(t_{k}\right)\right)\left[w\left(t_{k+1}\right)-w\left(t_{k}\right)\right]^{2}\right]
\end{aligned}
$$

Recall that for a Brownian process, $\mathbf{E}\left[w\left(t_{k+1}\right)-w\left(t_{k}\right)\right]^{2}=t_{k+1}-t_{k}$. As we take the limit as $t_{k+1}-t_{k} \rightarrow 0$, we can thus replace the $\left[w\left(t_{k+1}\right)-w\left(t_{k}\right)\right]^{2}$ term. Doing so and recognizing that the summations are by definition the Ito integral, we are left with

$$
\begin{aligned}
Y\left(t_{f}\right)-Y\left(t_{i}\right)= & \int_{t_{i}}^{t_{f}} f_{t}(t, X(t)) d t+\int_{t_{i}}^{t_{f}} f_{x}(t, X(t)) u(t) d t+\int_{t_{i}}^{t_{f}} f_{x}(t, X(t)) v(t) d w(t) \\
& +\frac{v\left(t_{k}\right)^{2}}{2} \int_{t_{i}}^{t_{f}} f_{x x}(t, X(t)) d t
\end{aligned}
$$

We have now shown Ito's Lemma for constant $u$ and $v$. It follows that for step functions, the identical applies, as they can be partitioned into finitely many constant functions over an interval. As shown in Lemma A.2 (in the appendix), it is possible to choose a sequence of step functions $u_{n}$ and $v_{n}$ so that

$$
\int_{t_{i}}^{t_{f}}\left|u(t)-u_{n}(t)\right| d t \rightarrow 0
$$




$$
\int_{t_{i}}^{t_{f}}\left|v(t)-v_{n}(t)\right|^{2} d t \rightarrow 0
$$

And $X_{n}(t)=X\left(t_{i}\right)+\int_{t_{i}}^{t} a_{n} d s+\int_{t_{i}}^{t} b_{n} d w(s)$ converges uniformly to $X(t)$ with probability 1 . As $f$ is continuously smooth, we can also say that $Y_{n}(t)=f\left(t, X_{n}(t)\right) \rightarrow Y(t)$ uniformly with probability 1 .

As $Y_{n}(t)$ is piecewise constant, we can say

$$
\begin{aligned}
Y\left(t_{f}\right)-Y\left(t_{i}\right)= & \int_{t_{i}}^{t_{f}}\left[f_{t}\left(t, X_{n}(t)\right)+f_{x}\left(t, X_{n}(t)\right) u_{n}(t)+\frac{1}{2} f_{x x}\left(t, X_{n}(t)\right) v_{n}{ }^{2}(t)\right] d t \\
& +\int_{t_{i}}^{t_{f}} f_{x}\left(t, X_{n}(t)\right) v_{n}(t) d w(t)
\end{aligned}
$$

As we let $n \rightarrow \infty$, we obtain Ito's Lemma in integral form,

$$
Y\left(t_{f}\right)-Y\left(t_{i}\right)=\int_{t_{i}}^{t_{f}}\left[f_{t}(t, X(t))+f_{x}(t, X(t)) u(t)+\frac{1}{2} f_{x x}(t, X(t)) v^{2}(t)\right] d t+\int_{t_{i}}^{t_{f}} f_{x}(t, X(t)) v(t) d w(t)
$$

The differential form of this is the theorem we set out to prove.

$$
s d Y(t)=\left[f_{t}(t, X(t))+f_{x}(t, X(t)) u(t)+\frac{1}{2} f_{x x}(t, X(t)) v^{2}(t)\right] d t+f_{x}(t, X(t)) v(t) d w(t)
$$

\subsection{Black-Scholes Equation}

\section{Financial Applications}

This brings us to the Black-Scholes equation for option pricing.

Consider a single stock, with price $S(t)$, which varies with time. Almgren argues that the value of the option deriving from that stock should have a market value that is a function of $S$ and $t$. Let us call this $D(t)=$ $V(t, S(t))$.

In the world of finance, the most significant descriptor of the profitability of an asset is its rate of return. In order to describe the pertrubations of the return on a share of stock, we will model it a geometric Brownian motion.

Definition A process takes on geometric (also known as exponential) Brownian motion if its logarithm follows a Brownian motion. In other words, only fractional changes take place as random variation. Its differential takes on the form

$$
d S=a S(t) d t+b S(t) d w(t)
$$

Where $a$ and $b$ are constants, and $w(t)$ is a Brownian motion.

Let the stock price take on a geometric Brownian motion, where the change in stock price is proportional to the current stock price, that is $d S=a S(t) d t+b S(t) d w(t)$. Note that by Ito's lemma,

$$
d D=\left[V_{t}+a S V_{S}+\frac{b^{2} S^{2}}{2} V_{S S}\right] d t+b S V_{S} d w(t)=\left[V_{t}+\frac{b^{2} S^{2}}{2} V_{S S}\right] d t+V_{S} d S
$$

Consider an investor, who holds a portfolio of the stock and its option,

$P(t)=N_{1}(t) S(t)+N_{2}(t) D(t)$.

The differential is

$$
d P=N_{1} d S+N_{2} d D=N_{1} d S+N_{2}\left[V_{t}+\frac{b^{2} S^{2}}{2} V_{S S}\right] d t+N_{2} V_{S} d S
$$

Malliaris[5] then makes the clever argument of holding a ratio of stock to derivative of $\frac{N_{1}}{N_{2}}=-V_{S}$ (this is known as a delta hedge), so that $N_{1} d S+N_{2} V_{S} d S=0$. We are left with

$$
d P=N_{2}\left[V_{t}+\frac{b^{2} S^{2}}{2} V_{S S}\right] d t
$$

Which is completely independent of Brownian motion (there is no $d w(t)$ term, explicit or implicit). As a result it can be considered "riskless". By the efficient market hypothesis, the return on this riskless asset must be equal to that on any other riskless asset, more specifically a government bond. Let the return on the government bond be $r(t)$. Then we have 


$$
\frac{d P}{P}=\frac{N_{2}\left[V_{t}+\frac{b^{2} S^{2}}{2} V_{S S}\right] d t}{N_{1} S+N_{2}}=r d t
$$

If we rearrange and normalize so that $N_{2}=1$, thus making $N_{1}=-V_{S}$, we get $\left[V_{t}+\frac{b^{2} S^{2}}{2} V_{S S}\right] d t=\left(-V_{S} S+V\right) r d t$ Or

$$
V_{t}(t, S)+\frac{b^{2} S^{2}}{2} V_{S S}(t, S)+S V_{S}(t, S)-r V(t, S)=0
$$

Which is the Black-Scholes differential equation for option pricing.

\section{Conclusion}

Almgren and Malliaris equally serve to make clear the link between stochastic processes and financial asset valuation and deepen the imminent provided in the beginning by Black and Scholes. The crux of the argument lies with Ito's lemma, which allows one to value an asset whose value is a random Brownian function of another asset. While Ito's original formula was developed for more scientific fields, it has found a position in financial analysis.

In their original thesis, Black and Scholes further solve their differential equation with condition that $V(t, S=0)=0$, and $F(T, S)=\max [0, S-E]$, where $T$ is the exercise date for the option, and $E$ is the exercise date indicated in the contract. As a result of the Black Scholes equation, the application of Stochastics to finance has been reinvigorated and today it has been applied to an overabundance of financial assets.
A Appendix
Lemma A.1 As $\max \left(t_{k+1}-t_{k}\right) \rightarrow 0$,
1. $\sum_{k=0}^{n-1}\left(t_{k+1}-t_{k}\right)^{2} \rightarrow 0$
2. $\quad \sum_{k=0}^{n-1}\left(t_{k+1}-t_{k}\right)\left(w\left(t_{k+1}\right)-w\left(t_{k}\right)\right) \rightarrow 0$
Proof

1. Without loss of generality, let $\left(t_{k+1}-t_{k}\right)$ be the largest partition of the space $\left[t_{i}, t_{f}\right]$. Since we are partitioning into $n$ segments, the average partition will have size $\frac{t_{f}-t_{i}}{n}$. Let our largest partition have size $c \frac{t_{f}-t_{i}}{n}$. Then

$$
\sum_{k=0}^{n-1}\left(t_{k+1}-t_{k}\right)^{2} \leq \sum_{k=0}^{n-1}\left(c \frac{t_{f}-t_{i}}{n}\right)^{2} \leq n\left(c \frac{t_{f}-t_{i}}{n}\right)^{2} \rightarrow 0
$$

As $\max \left(t_{k+1}-t_{k}\right) \rightarrow 0$, or equivalently as $n \rightarrow \infty$.

2. Similar to the previous example, note that

$$
\sum_{k=0}^{n-1}\left(t_{k+1}-t_{k}\right)\left(w\left(t_{k+1}\right)-w\left(t_{k}\right)\right) \leq c\left(t_{f}-t_{i}\right)\left(w\left(t_{k+1}\right)-w\left(t_{k}\right)\right)
$$

As a Brownian process is almost surely continuous, $c\left(t_{f}-t_{i}\right)\left(w\left(t_{k+1}\right)-w\left(t_{k}\right)\right) \rightarrow 0$ with probability 1 .

Lemma A.2 If $f(t)$ is in $\boldsymbol{M}[0, T]$, then there exists a sequence of step functions $f_{n}(t)$ in $\boldsymbol{M}[0, T]$, such that with probability 1 ,

\section{Proof}

$$
\lim _{n \rightarrow \infty} \int_{0}^{T}\left|f(t)-f_{n}(t)\right|^{2} d t=0
$$

Let us first consider a bounded function $g(t)$.

As it is bounded, at each point $t$, there is a sequence $g_{n}(t)$ to the value of $g(t)$ with probability 1 .

An arbitrary function in $\mathbf{M}[0, \mathrm{~T}]$ can be approximated by a bounded function to an arbitrary degree of accuracy. Thus, the sequence of step functions can also be approximated and are dense in the set of all functions.

\section{References}

[1] A. Malliaris. Ito's calculus in financial decision making. SIAM Review, 25(4):481-496, 1983

[2] K. Ito. On stochastic differential equations. Memoirs, American Mathematical Society, (4):1-51, 1951.

[3] I. Gikhman and A. Skorokhod. Introduction to the Theory of Random Processes. W. B. Saunders Company, 1969.

[4] F. Black and M. Scholes. The pricing of options and corporate liabilities. The Journal of Political Economy, 81(3):637-654, 1973.

[5] R. Almgren. Financial derivatives and partial differential equations. The American Mathematical Monthly, 109(1):1-12, 2002.

[6] J. C. Hull \& A. White, Journal of Finance, 42, (1987), 281

[7] J. C. Hull, Options, Futures \& Other Derivatives, Prentice Hall, (1997).

[8] R. C. Merton, Journal of Financial Economics, (1976), 125

[9] Paul Wilmott, Quantitative Finance, John Wiley, Chichester, (2000).

[10] A. Dragulescu, \& M. Yakovenko, Statistical Mechanics of money, income and wealth: A Short Survey, arXiv: cond-mat/0211175 v1, 9 Nov 2002 